\title{
Impact of Buprenorphine on Learning and Memory Ability Related to the Acetylcholinesterase Change in the Hippocampus of Rats
}

\author{
Mohammad Samini $^{1(\mathbb{D})}$, Tahereh Farkhondeh ${ }^{2,3} \mathbb{( D )}$, Mohsen Azimi-Nezhad ${ }^{4}(\mathbb{D}$, \\ Saeed Samarghandian 4 ,*(iD) \\ Faculty of Medicine, Mashhad University of Medical Sciences, Mashhad, Iran; saminim@mums.ac.ir (M.S.); \\ 2 Medical Toxicology and Drug Abuse Research Center (MTDRC), Birjand University of Medical Sciences (BUMS), \\ Birjand. Iran; farkhondeh2324@gmail.com (T.F.); \\ 3 Faculty of Pharmacy, Birjand University of Medical Sciences, Birjand, Iran \\ 4 Noncommunicable Diseases Research Center, Neyshabur University of Medical Sciences, Neyshabur. Iran; \\ aziminm@mums.ac.ir (M.A.N.); samarghandians1@ nums.ac.ir (S.S.); \\ * Correspondence: samarghandians1@nums.ac.ir;
}

Scopus Author ID 6507632790

Received: 5.01.2021; Revised: 31.01.2021; Accepted: 3.02.2021; Published: 7.02.2021

\begin{abstract}
Buprenorphine (BUP), a "synthetic opioid", may cause memory impairment. This investigation aimed to study the impact of BUP on memory function related to acetylcholinesterase (AChE) activity inhibition in male rats. 24 male Wistar rats were randomly divided into three groups; control (C) and two treatment groups BUP (0.3 and 1) ( $\mathrm{n}=8$, for each group). BUP (0.3 and $1 \mathrm{mg} / \mathrm{kg}$ ) was administrated subcutaneously once a day for 30 days. Normal saline $0.9 \%$ was injected in to control groups. In the end, animals were anesthetized and decapitated, and their hippocampus was dissected to assess AChE activity. There were no significant differences between the activities of AChE in the hippocampus in BUP-treated animals compared with controls. Besides, the activities of AChE in the BUP 0.3 group and BUP 1 group did not indicate a significant difference. These findings did not confirm the effect of BUP at doses of 0.3 and $1 \mathrm{mg} / \mathrm{kg}$ on memory function associated with the AChE activity inhibition.
\end{abstract}

Keywords: buprenorphine; memory; hippocampus; acetylcholinesterase.

(C) 2021 by the authors. This article is an open-access article distributed under the terms and conditions of the Creative Commons Attribution (CC BY) license (https://creativecommons.org/licenses/by/4.0/).

\section{Introduction}

Several studies indicated the role of cholinergic systems in memory function [1]. Acetylcholinesterase (AChE) is a target for ameliorating Alzheimer's disease (AD) symptoms [2]. Several animal studies have shown the inhibitory effect of some opioids, such as morphine [3]. However, there is some evidence related to cognitive impairment due to opioid administration in maintenance patients [3]. Buprenorphine (BUP), a "synthetic derivative of the thebaine" has been found as a relatively safe drug for the addicted patient [4]. The impact of BUP on the central nervous system has not been fully understood. The effect of opioids on the $\mu$-opioid receptors may lead to overexpression of these receptors and induce oxidative stress and inflammation in the hippocampus, leading to the disruption of memory function signaling pathways [5]. Besides, there is evidence related to the anti-acetylcholine esterase activity of opioids [6,7]. The acetylcholine accumulation in the synaptic cleft disturbs the nicotinic receptor function, resulting in several dysfunctions in the body, including memory impairment 
[8]. It has also been indicated that inhibition of $\mathrm{AChE}$ induced oxidative stress and caused neurotoxicity, evidenced by cognitive dysfunction in experimental animals [8]. However, the effect of opioids on the AChE activity in the brain was not thoroughly studied. Our study aimed to evaluate the effect of BUP on memory functions by measuring acetylcholine esterase activity in the hippocampus of rats.

\section{Materials and Methods}

24 male Wistar rats were randomly divided into the three groups as one control (C) and two treatment groups BUP (0.3 and 1$)$ ( $n=8$, for each group).

BUP $(0.3$ and $1 \mathrm{mg} / \mathrm{kg})$ was administrated subcutaneously once a day for 30 days. Normal saline $0.9 \%$ was injected in to control groups. In the end, animals were anesthetized with ketamine/ xylazine; lidocaine was also injected into the back of the neck. Then, the animals were decapitated, and their hippocampus was dissected and frozen in liquid N2. After thawing on ice, they were quickly mixed in phosphate buffer $\mathrm{pH} 7.4$ and centrifuged to separate supernatants for biochemical assay. The AChE activity was measured according to the colorimetric method that was described by Ellman et al. (1961) [9].

Data were analyzed by the InStat 3.0 program using one-way analysis of variance (ANOVA) and Tukey's tests. Data are shown as mean \pm SEM. The significant difference was $P<0.05$.

\section{Results and Discussion}

The levels of activity of AChE in the hippocampus in all groups have been indicated in Figure 1. As shown in figure 1, there were no significant differences between the activities of AChE in THE hippocampus in BUP-treated animals compared with controls. Also, the activities of AChE in the BUP 0.3 group and BUP 1 group did not indicate a significant difference.

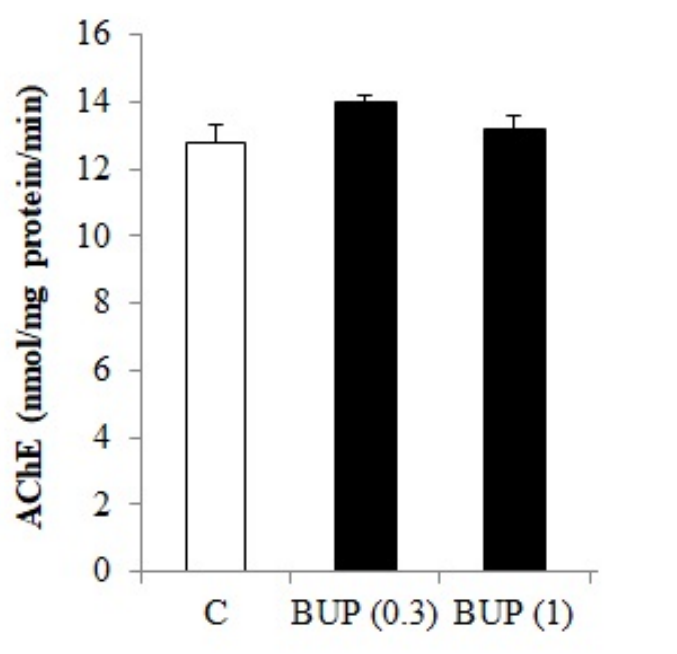

Figure 1. Effect of Buprenorphine (BUP) at dose $0 . .3$ and $1 \mathrm{mg} / \mathrm{kg}$ on AChE activity.

These findings showed that the BUP could not change the activity of AChE in the hippocampus of animals. Till now, BUP impact on the memory function has not been found. Clinical investigation has proposed that opioids can affect memory function depending on the severity of addiction and administration route [10]. Opioids can exert neurotoxic effects by induction oxidative stress. Oxidative stress is caused by an imbalance between oxidant/antioxidant content in the cells and damage to proteins, DNA, and lipids [11]. The 
brain's susceptibility to oxidative damage is very high due to its high levels of oxygen and polyunsaturated fatty acids and low antioxidant content. Oxidative stress has a vital role in cognitive impairment by a direct effect on neurons [12]. Hippocampus is the main region that memory function is mostly processed in this region of the brain. Besides, AChE is mostly secreted in the hippocampus [13-16]. Inhibition of AChE leads to an accumulation of acetylcholine in the synapse, leading to an elevation in oxidative stress in this region of the brain and disturbing the hippocampal function [8]. In normal physiological status, oxidative stress is regulated by antioxidant content. However, in abnormal physiological conditions, the antioxidants system cannot combat oxidative damage [17-20]. One of the main mechanisms involved in memory impairment is oxidative stress. Inhibition of the Ach activity may be one mechanism by which opioids inserts toxic effects [8]. Our findings could not show the effect of BUP at different doses $(0.3$ and $1 \mathrm{mg} / \mathrm{kg})$ on the AChE activity. This finding could not confirm or reject the effect of BUP on memory function through a change in the AChE activity due to the dose and time-dependent effect of opioids on the nervous system.

\section{Conclusions}

The present study did not indicate memory impairment through inhibition of AChE activity. It is better to design more studies to evaluate the effects of opioids on memory function through various AChE activity modification mechanisms.

\section{Funding}

This study was supported financially by the National Institute for Medical Research Development (NIMAD), Iran. Grant number: 963633.

\section{Acknowledgments}

The authors would like to thank the Research Affairs of the National Institute for Medical Research Development (NIMAD) for financially supporting this work.

\section{Conflicts of Interest}

The authors declare no conflict of interest.

\section{References}

1. Smach, M.A.; Hafsa, J.; Charfeddine, B.; Dridi, H.; Limem, K.; Jihene, B.A. Arthrophytum scoparium Extract Improves Memory Impairment and Affects Acetylcholinesterase Activity In Mice Brain. Curr. Pharm. Biotechnol. 2020, 21, 480-487, https://doi.org/10.2174/1389201020666191202142226.

2. Hulya, A.; İlhami, G. Potent Acetylcholinesterase Inhibitors: Potential Drugs for Alzheimer's Disease. MiniRev. Med. Chem. 2020, 20, 703-715, https://doi.org/10.2174/1389557520666200103100521.

3. Strand, M.C.; Vindenes, V.; Gjerde, H.; Mørland, J.G.; Ramaekers, J.G. A clinical trial on the acute effects of methadone and buprenorphine on actual driving and cognitive function of healthy volunteers. Br. J. Clin. Pharmacol. 2019, 85, 442-453, https://doi.org/10.1111/bcp.13818.

4. Yamamoto, T.; Shono, K.; Tanabe, S. Buprenorphine Activates $\mu$ and Opioid Receptor Like-1 Receptors Simultaneously, but the Analgesic Effect Is Mainly Mediated by $\mu$ Receptor Activation in the Rat Formalin Test. J. Pharmacol. Exp. Ther. 2006, 318, 206, https://doi.org/10.1124/jpet.105.100859.

5. Konijnenberg, C.; Melinder, A. Salivary cortisol levels relate to cognitive performance in children prenatally exposed to methadone or buprenorphine. Dev Psychobiol. 2020, 62(3), 409-418, doi: 10.1002/dev.21921.

6. Sim, M.K.; Chua, M.E. Inhibition of acetylcholinesterase by various opioids. Clin. Exp. Pharmacol. Physiol. 1986, 13, 159-162, https://doi.org/10.1111/j.1440-1681.1986.tb00330.x. 
7. Samarghandian S, Ohata H, Yamauchi N, Shibasaki T. Corticotropin-releasing factor as well as opioid and dopamine are involved in tail-pinch-induced food intake of rats. Neuroscience. 2003 Jan 31;116(2):519-24.

8. Bali, Y.A.; Kaikai, N.-e.; Ba-M'hamed, S.; Bennis, M. Learning and memory impairments associated to acetylcholinesterase inhibition and oxidative stress following glyphosate based-herbicide exposure in mice. Toxicology 2019, 415, 18-25, https://doi.org/10.1016/j.tox.2019.01.010.

9. Ellman, G.L.; Courtney, K.D.; Andres, V.; Featherstone, R.M. A new and rapid colorimetric determination of acetylcholinesterase activity. Biochem. Pharmacol. 1961, 7, 88-95, https://doi.org/10.1016/00062952(61)90145-9.

10. Baldacchino, A.; Tolomeo, S.; Balfour, D.J.; Matthews, K. Profiles of visuospatial memory dysfunction in opioid-exposed and dependent populations. Psychol. Med. 2019, 49, 1174-1184, https://doi.org/10.1017/S0033291718003318.

11. Samarghandian, S.; Azimi-Nezhad, M.; Afshari, R.; Farkhondeh, T.; Karimnezhad, F. Effects of Buprenorphine on Balance of Oxidant/Antioxidant System in the Different Ages of Male Rat Liver. $J$. Biochem. Mol. Toxicol. 2015, 29, 249-253, https://doi.org/10.1002/jbt.21691.

12. Mohamed, H.M.; Mahmoud, A.M. Chronic exposure to the opioid tramadol induces oxidative damage, inflammation and apoptosis, and alters cerebral monoamine neurotransmitters in rats. Biomed. Pharmacother. 2019, 110, 239-247, https://doi.org/10.1016/j.biopha.2018.11.141.

13. Cope, Z.A.; Lavadia, M.L.; Joosen, A.J.M.; van de Cappelle, C.J.A.; Lara, J.C.; Huval, A.; Kwiatkowski, M.K.; Picciotto, M.R.; Mineur, Y.S.; Dulcis, D.; Young, J.W. Converging evidence that short-active photoperiod increases acetylcholine signaling in the hippocampus. Cognitive, Affective, \& Behavioral Neuroscience 2020, 20, 1173-1183, https://doi.org/10.3758/s13415-020-00824-2.

14. Hussein, R.A.; Afifi, A.H.; Soliman, A.A.F.; El Shahid, Z.A.; Zoheir, K.M.A.; Mahmoud, K.M. Neuroprotective activity of Ulmus pumila L. in Alzheimer's disease in rats; role of neurotrophic factors. Heliyon 2020, 6, e05678, https://doi.org/10.1016/j.heliyon.2020.e05678.

15. Oliva, A.; Fernández-Ruiz, A.; Leroy, F.; Siegelbaum, S.A. Hippocampal CA2 sharp-wave ripples reactivate and promote social memory. Nature 2020, 587, 264-269, https://doi.org/10.1038/s41586-020-2758-y.

16. Yasui, B. Memory in the hippocampus. Med. Hypotheses 2019, 125, 8-9, https://doi.org/10.1016/j.mehy.2019.02.008.

17. Yamamura, H.; Suzuki, Y.; Asai, K.; Imaizumi, Y.; Yamamura, H. Oxidative stress facilitates cell death by inhibiting Orai1-mediated Ca2+ entry in brain capillary endothelial cells. Biochem. Biophys. Res. Commun. 2020, 523, 153-158, https://doi.org/10.1016/j.bbrc.2019.12.035.

18. Butterfield, D.A.; Halliwell, B. Oxidative stress, dysfunctional glucose metabolism and Alzheimer disease. Nature Reviews Neuroscience 2019, 20, 148-160, https://doi.org/10.1038/s41583-019-0132-6.

19. Gustavo, E.B.; Hector, A.S.; Sonia, J.M.; Laura, R.G. Oxidative Stress-Induced Brain Damage Triggered by Voluntary Ethanol Consumption during Adolescence: A Potential Target for Neuroprotection? Curr. Pharm. Des. 2019, 25, 4782-4790, https://doi.org/10.2174/1381612825666191209121735.

20. Alzoubi, K.H.; Al-Jamal, F.F.; Mahasneh, A.F. Cerebrolysin prevents sleep deprivation induced memory impairment and oxidative stress. Physiol. Behav. 2020, 217, 112823, https://doi.org/10.1016/j.physbeh.2020.112823. 